\title{
Training mid-career internists to perform high-quality colonoscopy: a pilot training programme to meet increasing demands for colonoscopy
}

\author{
Nicole K Shah-Ghassemzadeh, ${ }^{1}$ Christian S Jackson, ${ }^{2}$ David Juma, ${ }^{3}$ Richard M Strong ${ }^{2}$
}

'Department of Internal Medicine, Loma Linda University Medical Center, Loma Linda, California, USA

${ }^{2}$ Division of Gastroenterology, Loma Linda VA Medical Center, Loma Linda, California, USA ${ }^{3}$ School of Public Health, Loma Linda University Medical Center, Loma Linda, California, USA

\section{Correspondence to} Dr Nicole K Shah-

Ghassemzadeh, Loma Linda University Medical Center, Department of Internal Medicine, 11234 Anderson St. Rm 1511, Loma Linda, CA 92354, USA;

nshahghassemzadeh@|lu.edu

Received 11 October 2016 Revised 20 December 2016 Accepted 1 January 2017 Published Online First 19 January 2017

\section{ABSTRACT \\ Background Colorectal cancer $(C R C)$ is the second} leading cause of cancer deaths in the USA. Despite a recent rise in CRC screening there remains an increasing demand for colonoscopy, yet a limited supply of gastroenterologists who can meet this need.

Objective To determine if a mid-career general internist (GIN) could be trained to perform high-quality colonoscopes via an intensive training programme. Design A GIN trained 2-3 days/week, 4-5 hours/day, for 7 months with an experienced gastroenterologist. Their independent performance was then compared with that of a gastroenterology attending (GA), with and without a gastroenterology fellow (GF).

Main measures The primary outcome was to compare caecal intubation rates, adenoma detection rates (ADRs), interval $C R C$ rates and complications between the three groups.

Key results 989 patients were initially included in the study, and 818 were included in the final analysis. Caecal intubation rates were $95 \%, 94 \%$ and $93 \%$ for the GIN, GA+GF and GA, respectively $(p=0.31)$. The overall polyp detection rates were $68 \%, 39 \%$ and $44 \%$ among the GIN, GA+GF and GA, respectively $(p<0.0001)$. The ADRs were $56 \%, 33 \%$ and $34 \%$ for the GIN, GA+GF and GA, respectively $(p<0.0001)$. Three complications occurred, all within the GA group. No interval cancers were diagnosed within a 5-year surveillance period, across all three groups.

Conclusions The GIN attained high success rates in all quality measures. Training mid-career GINs to perform high-quality screening colonoscopes, through a standardised curriculum, may be a reasonable approach to address the growing demand for colonoscopists.

\section{BACKGROUND}

Colorectal cancer (CRC) is the third most common cancer, and the second leading cause of cancerrelated mortality in the USA. ${ }^{1}{ }^{2}$ It has been estimated that if the national guidelines for CRC screening were strictly followed, as many as $60 \%$ of deaths from CRC could be prevented. ${ }^{3}$ Colonoscopy is currently the most commonly used CRC screening modality within the USA, likely owing to its ability to provide complete visualisation of the colon and rectum, while simultaneously allowing for tissue sampling and polyp removal. ${ }^{4}$ Although the overall percentage of USA adults aged over 50 years who received CRC screening by either faecal occult blood test, sigmoidoscopy or colonoscopy nearly doubled from $34.8 \%$ in 1987 to $66.1 \%$ in 2010 , approximately $40 \%$ of Americans are still not receiving CRC screening according to the national guidelines. $^{3}$ 5 Furthermore, the lowest CRC screening prevalence rates have been consistently found among Hispanics, those with low socioeconomic status, those with a maximum educational level of high school and the uninsured. ${ }^{6}$ CRC screening compliance is another factor that impacts screening rates, and is significantly driven by patients' fears of complications and procedure-induced pain, which are ultimately related to the endoscopists' level of clinical training and experience. ${ }^{8}$

Diagnostic resources for CRC screening are expected to decrease within the USA. ${ }^{9}$ With the growing size of the elderly population, a deficiency of 1050-1550 gastroenterologists is anticipated by 2020, therefore creating a significant need for welltrained endoscopists. ${ }^{3}$ At the present time, 400 gastroenterologists complete training programmes yearly, which already fails to meet the current demand for high-quality CRC screening. ${ }^{10}$ In addition, with the advent of the Patient Protection and Affordable Care Act, expansion of insurance coverage is expected to increase CRC screening rates. ${ }^{11}$ To address these demands, it has been estimated that gastroenterology training programmes will need to increase their capacity by at least one-third. ${ }^{11}$

In the USA, $33 \%$ of a total 1.6 million screening colonoscopes are performed by non-gastroenterologists. ${ }^{12}$ Historically, colonoscopy training has not been a focus of traditional primary internal medicine training, as it has been in the disciplines of family practice and general surgery. ${ }^{13}$ Colonoscopy is a complex technical procedure requiring substantial training, with significant medicolegal risks. ${ }^{14-18}$ CRC-related litigation is on the rise with medicolegal risks primarily encompassing failure to follow screening guidelines, poor-quality examinations leading to missed lesions, failure to systematically document and track outcomes as well as failure to maintain appropriate follow-up testing. The quality indicators for CRC screening colonoscopy that are currently recommended by the American Society for Gastrointestinal Endoscopy (ASGE) include a caecal intubation rate $\geq 95 \%$, an adenoma detection rate (ADRs) $\geq 20 \%$ in women and $\geq 30 \%$ in men aged $\geq 50$ years, a mean total withdrawal time $\geq 6 \mathrm{~min}$, a perforation rate of $1 / 1000$ and a postpolypectomy bleeding rate of $<1 \%{ }^{19}$ While some studies have shown that nongastroenterologists have a higher risk of interval 
cancers, multiple studies have demonstrated that primary care physicians performing screening colonoscopes are in fact meeting the ASGE's quality indicators. ${ }^{20-23}$

CRC screening is highly dependent on the competence of the endoscopist. ${ }^{24}$ Multiple studies have demonstrated that the role of the endoscopist has a more significant effect on ADRs and interval CRC rates than the age or gender of the patient. ${ }^{25-27}$ Based on the aforementioned evidence and a waitlist of patients needing diagnostic and screening colonoscopy at our facility, we felt this was a prime opportunity to determine if a mid-career general internist (GIN), with previous experience performing flexible sigmoidoscopy, could be trained via an intensive programme to perform high-quality colonoscopes. We describe our experience with a feasibility pilot programme within the Loma Linda Veterans Affairs (VA) Healthcare System.

\section{DESIGN}

A mid-career GIN who had previous experience performing $>1700$ flexible sigmoidoscopes was selected to participate in our intensive colonoscopy training programme. The GIN spent 4-5 hours/day, 2-3 days/week training with a supervising staff gastroenterologist, for a total of 7 months, during which a $180 \mathrm{~cm}$ adult colonoscope (Pentax, Montavale, New Jersey, USA) was used in all cases. Video simulators as well as porcine colon models were also used to practice polypectomy techniques on flat, sessile and pedunculated polyps. After the 7-month training period was completed, the internist then performed only colonoscopes independently for an additional 7-month period. All procedures performed by the GIN, gastroenterology attending (GA) and GA+ gastroenterology fellow (GF) were allocated from a general endoscopy list. A consult for colonoscopy was sent to the gastroenterology department. The patient was either seen in clinic or in a nurse education class to determine the indication for colonoscopy and the appropriate precolonoscopy preparation. The patient was then scheduled for colonoscopy and placed on the aforementioned list. The presence of anastomosis, unusual anatomy and/or inadequate sedation were criteria for exclusion from the analysis as these barriers to a complete and thorough colonoscopic exam prohibit the acquisition of conclusive data about the presence of adenomas, which would ultimately affect one of the primary outcomes of the study, namely ADR.

\section{MAIN MEASURES}

The GIN's performance during the post-training period was evaluated monthly based on the following colonoscopy findings: caecal and terminal ileum (TI) intubation rates, overall polyp detection rates, ADR, withdrawal time, quality of withdrawal and number of complications. We also compared caecal and TI intubation rates, overall polyp detection rates, ADR and hyperplastic polyp detection rates among experienced gastroenterology attendings (GA) performing colonoscopy both independently and with first through third year GF. To compare patient's baseline characteristics, either an analysis of variance or a $\chi^{2}$ test was used. A $\chi^{2}$ test was used to compare the colonoscopy findings between the three groups. To account for low cell counts in the occurrence of colonoscopy complications, Fisher's exact test was used. The analysis was conducted using SAS V.9.4. This study was approved by the Institutional Review Board of the Loma Linda VA Healthcare System.

\section{RESULTS}

The ability of the GIN to perform independent colonoscopy was defined by the comfort of the supervising GA, which was defined by caecal intubation in $>95 \%$ of all cases, without the need for rescue. After successfully completing the pilot training programme, the GIN was granted permission to begin performing independent colonoscopes. Our analysis reflects the GIN's independent performance. The GIN performed a total of 381 colonoscopes independently over a 7 -month period. A total of 61 cases were excluded from the analysis due to poor preparation (32), abnormal anatomy (26), inadequate sedation (1) and incomplete information to determine the indication for colonoscopy (2). A total of 320 patients were analysed, with a mean age of $63.8 \pm 10.6$ years, and a mean body mass index of (BMI) 30.2 $\pm 6.1 \mathrm{~kg} / \mathrm{m}^{2}$. The predominant race among these patients was Caucasian (61\%), and the majority of patients were male (90\%) (table 1).

During the same time period, GA+GF performed 103 colonoscopes, 21 of which were excluded from the analysis for poor preparation (18), abnormal anatomy or anastomosis (2) and incomplete information (1). GA also independently performed 505 colonoscopes. Eighty-nine of these cases were excluded from the analysis due to poor preparation (67), presence of an anastomosis (17), inadequate sedation (2) and incomplete information (3). The mean age, BMI and racial breakdown of these patients were similar to that of the GIN group. The baseline characteristics of all patients within the three different groups are summarised in table 1 . The primary indications for colonoscopy among all three groups included surveillance (30\%), bleeding (16\%), anaemia (13\%), occult positive stools $(13 \%)$ and screening (10\%) (table 2). There was no statistically significant difference in the indications for colonoscopy among the three study groups $(\mathrm{p}=0.341)$.

Of 320 colonoscopes, the GIN reached the caecum with appropriate landmark identification in 305 cases (95\%), compared with $94 \%$ for GF+GA and $93 \%$ for GA alone $(\mathrm{p}=0.31)$. The GIN reached the TI in $47 \%$ of cases, compared with $32 \%$ for the GF+GA and $35 \%$ for the GA $(\mathrm{p}=0.0008)$. The overall polyp detection rates were $68 \%, 39 \%$ and $44 \%$ among the GIN, GF+GA and GA, respectively $(p<0.0001)$. When the size of the polyps detected were categorised as $<5 \mathrm{~mm}, 5 \mathrm{~mm}-$ $1 \mathrm{~cm}$ and $>1 \mathrm{~cm}$ and then compared among the three study groups, there was no statistically significant difference in polyp size $(\mathrm{p}=0.066)$. The ADR was $56 \%$ for the GIN versus $33 \%$ for the GF+GA and $34 \%$ for the GA $(\mathrm{p}<0.0001)$. Hyperplastic polyps were found in $27 \%$ of the cases for the GIN, $17 \%$ for $\mathrm{GF}+\mathrm{GA}$ and $15 \%$ for the GA alone $(\mathrm{p}=0.0003)$. High-risk polyps were found in $23 \%$ of colonoscopes performed by GIN, $22 \%$ for GF+GA and $16 \%$ for the GA $(p=0.047)$. Withdrawal time was $>6$ min for all colonoscopes performed during the 7 -month period for all three groups. There were a total of three complications that occurred within the study period, all of which were GA's cases, and consisted of postpolypectomy bleeding. There were no bowel perforations among all three groups. The aforementioned findings are summarised in table 3.

All colonoscopes were reviewed retrospectively to determine the interval CRC rate. There were no interval CRCs diagnosed in all three groups during the 5 -year surveillance period.

\section{DISCUSSION}

Among the recommended CRC screening modalities, colonoscopy is widely preferred and considered the gold standard given the advantage of cancer prevention via removal of adenomatous polyps. ${ }^{45}$ This has been well established by the findings in the National Polyp Study. ${ }^{28}$ A 2012 follow-up study aimed at evaluating the long-term effects of colonoscopic polypectomy on CRC-related mortality further demonstrated that polypectomy 
Table 1 Patient's baseline characteristics

\begin{tabular}{|c|c|c|c|c|c|}
\hline & GIN & $\mathrm{GF}+\mathrm{GA}$ & GA & Total & p Value \\
\hline Age (mean, range) & $63.8 \pm 10.6,27-91$ & $63.6 \pm 11.8,32-96$ & $62.7 \pm 11.2,26-89$ & $63.2 \pm 11.0,26-96$ & 0.40 \\
\hline BMI (mean, range) & $30.2 \pm 6.1,16-60$ & $29.7 \pm 7.5,15-52$ & $30.3 \pm 6.4,14-52$ & $30.2 \pm 6.4,14-60$ & 0.72 \\
\hline \multicolumn{6}{|l|}{ Gender (N, \%) } \\
\hline Male & $287(90)$ & $72(88)$ & $375(90)$ & $734(90)$ & 0.82 \\
\hline \multicolumn{6}{|l|}{ Ethnicity (N, \%) } \\
\hline Caucasian & $196(61)$ & $48(59)$ & $232(56)$ & $476(58)$ & 0.01 \\
\hline Hispanic & $24(8)$ & 12 (15) & $67(16)$ & $103(13)$ & \\
\hline African-American & $40(13)$ & $14(17)$ & $47(11)$ & $101(12)$ & \\
\hline Asian & $4(1)$ & $0(0)$ & $6(1)$ & $10(1)$ & \\
\hline American Indian/Alaska native & $5(2)$ & $1(1)$ & $4(1)$ & $10(1)$ & \\
\hline Pacific Islander & $8(3)$ & $0(0)$ & $12(3)$ & $20(2)$ & \\
\hline Unknown/mixed & $37(12)$ & $6(7)$ & $36(9)$ & $79(10)$ & \\
\hline Declined & $6(2)$ & $1(1)$ & $12(3)$ & $19(2)$ & \\
\hline
\end{tabular}

GA, gastroenterology attending; GF, gastroenterology fellow; GIN, general internist.

reduced CRC-associated mortality by $53 \% .{ }^{28}$ Colonoscopy has also been shown to be a more sensitive tool in detecting adenomas when compared with faecal immunochemical testing. ${ }^{29}$ These findings add to the body of literature endorsing colonoscopy as a highly recommended modality for CRC screening in the USA.

Unfortunately, the prospect of delivering screening and diagnostic colonoscopes to all patients in need will likely be unattainable without an increase in the number of GI fellowship training positions. Despite this demand, fellowship training programmes have actually reduced the number of training positions based on older assessments of anticipated gastroenterology needs. ${ }^{30}$ Re-evaluation of these data more recently has suggested that 32500 gastroenterologists would be needed if a screening colonoscopy was performed every 10 years for the general population. ${ }^{12}$ This number would considerably increase if diagnostic colonoscopes were also taken into account.

Based on the findings in our study, we believe that training a mid-career GIN to perform screening and diagnostic colonoscopes may be a reasonable option to help address this growing problem. Considering that there was no significant difference in the indications for colonoscopy among the three groups, the GIN in this study attained a high success rate in all colonoscopy

Table 2 Indications for colonoscopy

\begin{tabular}{lll}
\hline Indications for colonoscopy & $\begin{array}{l}\text { Number of } \\
\text { patients }\end{array}$ & $\begin{array}{l}\text { Percentage of } \\
\text { patients }\end{array}$ \\
\hline Surveillance & 254 & 30 \\
Bleeding & 130 & 16 \\
Anaemia & 107 & 13 \\
Occult positive & 102 & 13 \\
Screening & 84 & 10 \\
Change in bowel habits & 53 & 7 \\
Flexible sigmoidoscopy or & 52 & 6 \\
radiographic findings & & \\
Other symptoms & 28 & 3 \\
Inflammation & 14 & 2 \\
Total & $815^{*}$ & \\
\hline
\end{tabular}

*The indication for colonoscopy was missing for three patients.

tIncludes abdominal pain, diverticular disease, concern for fistula, evaluation for colostomy reversal, decompression, polyp removal, weight loss and pelvic mass. quality measures as recommended by the ASGE, after just 7 months of training. In fact, the GIN in our study actually outperformed the GA and GA+GF among all of our quality parameters. This was statistically significant with regard to TI intubation rates, overall polyp detection rates, ADRs as well as the rates of hyperplastic and high-risk polyp detection. However, it should be noted that the higher rate of TI intubation by the GIN compared with the other cohorts likely reflects a matter of process rather than indication or necessity, as only a total of 76 patients in the study had indications for TI intubation specifically, namely chronic diarrhoea and inflammatory bowel disease. Among our study population, there were actually more patients in the GA and GA+GF cohorts who warranted TI intubation, compared with the GIN group alone.

In addition, there were no interval CRCs diagnosed throughout the 5-year surveillance period. It is thought that certain markers of high-quality colonoscopy such as ADR, reflect a meticulously performed procedure that ensures both small and large polyps are detected and removed, we feel that a meticulous examination can be performed by mid-career GIN who is trained by an experienced gastroenterologist. The idea that a mid-career GIN can be trained to perform colonoscopes at the level of a seasoned gastroenterologist within a reasonable timeframe, has positive implications for the nation's growing need for more qualified colonoscopists. Our findings are similar to those described in a recently published study by McClellan

Table 3 Colonoscopy findings $(n=818)$

\begin{tabular}{lccccc}
\hline & $\begin{array}{l}\text { GIN (N, } \\
\%)\end{array}$ & $\begin{array}{l}\text { GF+GA } \\
(\mathbf{N}, \%)\end{array}$ & $\begin{array}{l}\text { GA (N, } \\
\%)\end{array}$ & $\begin{array}{l}\text { Total (N, } \\
\%)\end{array}$ & p Value \\
\hline Caecum rate & $305(95)$ & $77(94)$ & $385(93)$ & $767(94)$ & 0.31 \\
TI rate & $151(47)$ & $26(32)$ & $144(35)$ & $321(39)$ & $<0.001$ \\
Any polyps & $218(68)$ & $32(39)$ & $181(44)$ & $431(53)$ & $<0.001$ \\
Adenomas & $179(56)$ & $27(33)$ & $143(34)$ & $349(43)$ & $<0.001$ \\
Hyperplastic polyp & $87(27)$ & $14(17)$ & $63(15)$ & $164(20)$ & $<0.001$ \\
High-risk polyps & $72(23)$ & $18(22)$ & $65(16)$ & $155(19)$ & $0.047^{*}$ \\
Complications & $0(0)$ & $0(0)$ & $3(0.7)$ & $3(0.36)$ & $0.46 \dagger$
\end{tabular}

* The significance was 0.91 between GF and GIN, 0.16 between GF and GA, 0.02 between GA and GIN.

tFisher's exact test.

GA, gastroenterology attending; GF, gastroenterology fellow; GIN, general internist; $\mathrm{TI}$, terminal ileum. 
et $a l,{ }^{20}$ evaluating the quality of colonoscopes performed by family medicine physicians.

Despite the positive implications of our findings, they do not imply that general internists who are trained to perform colonoscopy should replace gastroenterologists. Rather, in attempts to address the growing need for more certified colonoscopists, we support the concept of an intensive colonoscopy training programme of at least 7 months for mid-career primary care physicians, with oversight from an experienced gastroenterologist. Such training will potentially allow for more timely screening and diagnostic colonoscopes. The colonoscopy-trained internist would also serve well in rural, underserved areas, as has been demonstrated in South Carolina where colonoscopy-trained primary care physicians have helped provide screening colonoscopes to African-Americans, who are known to have higher CRC rates compared with Caucasians. ${ }^{31}$

The main predictor of delayed-stage CRC in California between 2004 and 2008 was socioeconomic status, with the majority of delayed-stage CRCs found in the right (proximal) colon, an area that is inaccessible with the flexible sigmoidoscopy. ${ }^{32}$ Investigators have reported that individuals of lower economic status are less likely to be screened and are in general screened differently. ${ }^{33-35}$ Conversely, areas with a higher density of gastroenterologists have been shown to have lower incidences of CRC and specifically late-stage CRC. ${ }^{36}$ This disparity could potentially be addressed by colonoscopy-trained primary care physicians.

This study has several limitations. First, this is a single-centre, non-randomised, prospective study performed at a Department of Veterans Affairs Healthcare System, which is known to have a male predominance, as well as a high prevalence of adenomas and advanced colorectal neoplasia. ${ }^{37}$ Our training programme included only one mid-career GIN who was highly motivated, and had considerable experience in flexible sigmoidoscopy prior to the study. Early in her practice, the GIN in our study learned how to perform flexible sigmoidoscopy under the instruction of a seasoned gastroenterologist with whom she trained for several months. After this initial training, she helped facilitate a general internal medicine run sigmoidoscopy clinic, where she continued to perform flexible sigmoidoscopes for 2-3 years before undertaking our pilot colonoscopy training programme. Admittedly, the time our GIN invested in our 7-month training programme before she was permitted to perform colonoscopes independently may not be a feasible time commitment for most practicing primary care physicians. In addition, when the internist in this study performed colonoscopes independently, she performed no more than four colonoscopes within a 4-hour period, which is generally not the typical schedule for a practicing gastroenterologist. Lastly, we did not extract pathology data regarding whether excision of the polyps detected and treated was determined to be complete excision or not, and therefore cannot compare complete polyp excision rates among the three study groups.

\section{CONCLUSION}

Based on our positive experience in this study, along with previously published data with similar findings, ${ }^{20} 22$ we support the concept of a standardised programme to train nongastroenterologists how to perform high-quality and safe screening colonoscopes under the direction of an experienced gastroenterologist. Furthermore, experienced gastroenterologists are best prepared to develop such a curriculum while providing continued evaluation and oversight over mid-career primary care physicians throughout their training.

\section{Main messages}

- Colonoscopy is the gold standard for colorectal cancer (CRC) screening; however, there remains a shortage of capable endoscopists to meet the growing demands for screening colonoscopes within the USA.

- The quality indicators for CRC screening colonoscopy per the American Society for Gastrointestinal Endoscopy (ASGE) include a caecal intubation rate $\geq 95 \%$, an adenoma detection rate (ADRs) $\geq 20 \%$ in women and $\geq 30 \%$ in men aged $\geq 50$ years, a mean total withdrawal time $\geq 6 \mathrm{~min}$, a perforation rate of $1 / 1000$ and a postpolypectomy bleeding rate of $<1 \%$.

- The general internist in this study attained a high success rate in all colonoscopy quality measures as recommended by the ASGE, after 7 months of training with an experienced gastroenterologist.

- General internists can be trained to perform high-quality colonoscopy as a means to help eradicate the disparities in access to CRC screening within the USA.

\section{Current research questions}

- What are the essentials of creating a standardised colonoscopy training programme aimed towards instructing general internists how to perform high-quality colonoscopy?

- Is it financially feasible to create a nationally standardised colonoscopy training programme for non-gastroenterologists?

- What criteria should be used to select general internists as participants in a nationally standardised colonoscopy training programme?

- For general internists who are trained to perform high-quality colonoscopy, what are the reasonable limitations that should be set regarding their management of various colonoscopy findings?

Contributors Study concept/design, acquisition of the data, review and editing of the manuscript: CSJ (Loma Linda Veterans Affairs (VA) Medical Center, Division of Gastroenterology). Study concept/design and critical review of the manuscript: RMS (Loma Linda VA Medical Center, Division of Gastroenterology). Interpretation of data and drafting of the manuscript: NKS-G (Loma Linda University Medical Center, Department of Internal Medicine). Data analysis and interpretation of data: DJ (Loma Linda University School of Public Health).

Competing interests CSJ is a speaker for Medivators.

Ethics approval Institutional Review Board of the Loma Linda Veterans Affairs Healthcare System.

Provenance and peer review Not commissioned; externally peer reviewed.

\section{REFERENCES}

1 Edwards BK, Noone AM, Mariotto $A B$, et al. Annual report to the nation on the status of cancer, 1975-2010, featuring prevalence of comorbidity and impact on survival among persons with lung, colorectal, breast, or prostate cancer. Cancer 2014;120:1290-314.

2 Haggar FA, Boushey RP. Colorectal cancer epidemiology: incidence, mortality survival, and risk factors. Clin Colon Rectal Surg 2009;22:191-7.

3 http://www.olympusamerica.com/corporate/docs/Lewin-Gastroenterologist-Report.pdf (accessed Dec 2015)

4 Garborg K. Colorectal cancer screening. Surg Clin N Am 2015;95:979-89. 
5 Yang DX, Gross CP, Soulos PR, et al. Estimating the magnitude of colorectal cancers prevented during the era of screening: 1976 to 2009. Cancer 2014;120:2893-901.

6 Center for Disease Control and Prevention. Morbidity and Mortality Weekly Report (7/9/2010). http://www.cdc.gov/mmwr/preview/mmwrhtml/mm5926a3.htm (accessed 15 Dec 2015).

7 Center for Disease Control and Prevention. Morbidity and Mortality Weekly Report (11/22/2013). http://www.cdc.gov/mmwr/pdf/other/su6203.pdf (accessed 16 Dec 2015).

8 Kapidzic A, Grobbee EJ, Hol L, et al. Attendance and yield over three rounds of population-based fecal immunochemical test screening. Am J Gastroenterol 2014;109:1257-64.

9 Brown ML, Klabunde CN, Mysliwiec P. Current capacity for endoscopic colorectal cancer screening in the United States: data from The National Cancer Institute Survey of Colorectal Cancer Screening Practices. Am J Med 2003;115:129-33.

10 Wilcox CM. Are we on track for education and training of future gastroenterologists? Interview by Paul C Adams. Can J Gastroenterol 2009;23:794-5.

11 Cooper RA. Myth and reality underlying the needed expansion of graduate medical education. Gastroenterology 2009;136:2045-7.

12 Vijan S, Inadomi J, Hayward RA, et al. Projections of demand and capacity for colonoscopy related to increasing rates of colorectal cancer screening in the United States. Aliment Pharmacol Ther 2004;20:507-15.

13 Wilkins $T$, Jester $D$, Kenrick J, et al. The current state of colonoscopy training in family medicine residency programs. Fam Med 2004;36:407-11.

14 Rex DK, Kahi CJ, Levin B, et al. Guidelines for colonoscopy surveillance after cance resection: a consensus update by the American Cancer Society and US Multi-Society Task Force on Colorectal Cancer. CA Cancer J Clin 2006;56:160-7; quiz 185-6.

15 Rex DK, Bond JH, Winawer S, et al. Quality in the technical performance of colonoscopy and the continuous quality improvement process for colonoscopy: recommendations of the U.S. Multi-Society Task Force on Colorectal Cancer. Am J Gastroenterol 2002;97:1296-308.

16 Feld AD. Malpractice risks associated with colon cancer and inflammatory bowel disease. Am J Gastroenterol 2004:99:1641-4.

17 Feld AD. Medicolegal implications of colon cancer screening. Gastrointest Endosc Clin N Am 2002:12:171-9, viii-ix.

18 Rex DK, Bond JH, Feld AD. Medical-legal risks of incident cancers after clearing colonoscopy. Am J Gastroentrol 2001;96:952-7.

19 Rex DK, Schoenfeld PS, Cohen J, et al. Quality indicators for colonoscopy. Gastrointestinal Endosc 2015;81:31-53.

20 McClellan DA, Ojinnaka CO, Pope R, et al. Expanding access to colorectal cancer screening: benchmarking quality indicators in a primary care colonoscopy program. J Am Board Fam Med 2015;28:713-21.
21 Rabeneck L, Paszat LF, Saskin R. Endoscopic specialty is associated with incident colorectal cancer after a negative colonoscopy. Clin Gastroenterol Hepatol 2010;8:275-9.

22 Wilkins $\mathrm{T}$, Le Clair B, Smolkin $\mathrm{M}$, et al. Screening colonoscopes by primary care physicians: a meta-analysis. Ann Family Med 2009;7:56-62.

23 Singh $\mathrm{H}$, Penfold RB, De Coster C, et al. Predictors of serious complications associated with lower gastrointestinal endoscopy in a major city-wide health region. Can J Gastroenterol 2010:24:425-30.

24 Rogart JN, Siddiqui UD, Jamidar PA, et al. Fellow involvement may increase adenoma detection rates during colonoscopy. Am J Gastroenterol 2008;103:2841-6.

25 Rex DK. Maximizing detection of adenomas and cancers during colonoscopy. Am J Gastroenterol 2006;101:2866-77.

26 Brenner $\mathrm{H}$, Chang-Claude J, Seiler CM, et al. Protection from colorectal cancer after colonoscopy: a population-based, case-control study. Ann Intern Med 2011;154:22-30.

27 Kaminski MF, Regula J, Kraszewska E, et al. Quality indicators for colonoscopy and the risk of interval cancer. N Engl J Med 2010;362:1795-803.

28 Zauber AG, Winawer SJ, O'Brien MJ, et al. Colonoscopic polypectomy and long-term prevention of colorectal-cancer deaths. N Engl J Med 2012;366:687-96.

29 Quintero E, Castells A, Bujanda L, et al. Colonoscopy versus fecal immunochemical testing in colorectal-cancer screening. N Engl J Med 2012;366:697-706.

30 Meyer GS, Jacoby I, Krakauer $\mathrm{H}$, et al. Gastroenterolgy workforce modeling. JAMA 1996:276:689-94.

31 Lloyd SC, Harvey NR, Herbert JR, et al. Racial disparities in colon cancer. Primary care endoscopy as a tool to increase screening rates among minority patients. Cancer 2007;109:378-85.

32 Morgan JW, Cho MM, Guenzi CD, et al. Predictors of delayed-stage colorectal cancer: are we neglecting critical demographic information? Ann Epidemiol 2011;21: 914-21.

33 Beydoun HA, Beydoun MA. Predictors of colorectal cancer screening behaviors among average-risk older adults in the United States. Cancer Causes Control 2008;19:339-59.

34 Mandelblatt J, Andrews $\mathrm{H}, \mathrm{Kao} \mathrm{R}$, et al. The late-stage diagnosis of colorectal cancer: demographic and socioeconomic factors. Am J Public Health 1996;86:1794-7.

35 American Cancer Society. 2011. California Cancer Facts \& Figures. http://www. ccrcal.org/pdf/Reports/ACS_2011.pdf (accessed 16 Dec 2015).

36 Ananthakrishnan AN, Hoffmann RG, Saeian K. Higher physician density is associated with lower incidence of late-stage colorectal cancer. J Gen Intern Med 2010;25:1164-71.

37 Lieberman DA, Weiss DG, Bond JH, et al. Use of colonoscopy to screen asymptomatic adults for colorectal cancer. Veterans Affairs Cooperative Study Group 380. N Engl J Med 2000;343:162-8. 